\title{
Artificial Inverse Opal Structures for Sensing Applications
}

\author{
Xia Wu ${ }^{1}$, Sabrina Amrehn ${ }^{1}$, Xuyang Zhang ${ }^{1}$, Christian Schumacher ${ }^{2}$, Thorsten Wagner ${ }^{*}, 1$ \\ ${ }^{1}$ Department of Chemistry, University of Paderborn, Warburger Straße 100, D-33098, Germany, \\ thorsten.wagner@upb.de \\ 2 Direct Manufacturing Research Center (DMRC), University of Paderborn, Warburger Straße 100, \\ D-33098, Germany
}

\begin{abstract}
:
Optical sensing using photonic crystals (PhCs) as transducing materials is an emerging field in the gas and liquid sensor research. There is a wide range of properties of the PhCs that can be utilized to gain information about the pore fluid (gas/liquid). Identifying new sensing mechanisms is therefore one major topic in current research. Concepts are based on detection of e.g. refractive index changes in the pore fluid, structural changes in the $\mathrm{PhC}$ or optical changes caused by electronic interaction of the material with the target species. Therefore, irrespective of the details of the mechanism, elaborate material design is required. In this regard artificial inverse opal films synthesized by nano-casting methods are especially interesting because of their ease of fabrication and the variety of possible materials that can be structured. As an example $\mathrm{WO}_{3}$ inverse opal films fabricated by a simple nanocasting process and its application as sensing material in refractive index sensor for fluid detection are presented. A combination of theoretical considerations and experimental investigations leading to some general recommendations for the design of this type of sensor are given. For the presented fluid sensor, limit of detection of 0.001 refractive index unit (RIU) can be achieved. Also the thermal stability of the $\mathrm{WO}_{3}$ films will also be addressed briefly since it is crucial for potential high temperature $\left(>500^{\circ} \mathrm{C}\right)$ applications.
\end{abstract}

Key words: inverse opal, photonic crystal, refractive index sensor, fluid sensor, thermal stability

\section{Introduction}

Opal is a type of gemstone that displays iridescent colors. This attractive optical property is generated by the physical interaction between light and the periodically ordered nanometer-sized silica spheres that forms this natural material. These spheres are arranged in a 3D lattice structure similar to the crystal structure of hexagonal close-packed or face centered cubic materials (see the blue sphere model in Fig. 1), but in the length scale of the wavelength of visible light. This type of materials is known as photonic crystals (PhCs) which form photonic band gaps achieved by spatial modulations of refractive index. The constructive interference of light resulting from its periodic structure leads to the selective reflection of light of certain frequency within the band gaps. This photonic effect in visible spectrum is similar to the Bragg diffraction of $X$ ray from classical crystals formed by atoms or molecules.

Inverse opal structure is the negative replica of the opal structure, in which the solid spheres are replaced by air phase forming pores whereas the space between spheres is filled with a new material (see the red model in Fig. 1). Similar to the opal structure, inverse opal structure is also a 3D PhC. Its optical properties can be controlled by designing its photonic band structure which is in turn mainly controlled by the refractive index contrast between the material in the pore and the solid skeleton.

Artificial inverse opals as a type of PhCs are attractive sensing materials for gas/liquid sensors. The macroporous structure provides large and accessible specific surface area for the interaction between the analytes and its constituting material. For high temperature applications, it is expected to offer higher thermal stability comparing to its mesoporous counterparts. Its optical properties are often influenced by the refractive index contrast between the sensing material and the analytes or the physical interaction between these two. Therefore inverse opal structures can provide new sensing mechanisms with the advantage of remote sensing via optical readouts. Detailed overview of the research in this field can be found in reviews elsewhere [1,2]. 
In this proceeding, we first introduce a universally applicable but rather simple synthetic method for metal oxide inverse opal. In the second part, we show an application example of this inverse opal structure as a refractive index sensor for fluid detection. In the end, we address the thermal stability issue of the metal oxide inverse opal base on our preliminary results.

\section{Synthesis and Structure of Inverse Opal}

We fabricated the tungsten oxide $\left(\mathrm{WO}_{3}\right)$ inverse opal films in a two-step structure replication (nano-casting) process (see e.g. [3]). In the first step, we synthesized polymethylmethacrylate (PMMA) spheres used for forming the artificial opal template by surfactant-free emulsion polymerization [4,5]. These spheres were deposited onto glass substrates by ultrasonication facilitated sedimentation method which can result in uniform opal films with large ordered domains as shown in literature [6]. In the second step, we infiltrated the pores between the PMMA spheres with a metal oxide precursor (ammonium metatungstate hydrate). We then dried this composite at $60^{\circ} \mathrm{C}$, and thermally converted the precursor to metal oxide at $400{ }^{\circ} \mathrm{C}$ with simultaneous removal of the PMMA by combustion. The resulting structure is the $\mathrm{WO}_{3}$ inverse opal structure which is essentially a negative replica of the opal template. A scheme of this process is shown in Fig.1, and the detailed description of the preparation method can be found in elsewhere [5].

The size and quality of the spherical PMMA particles strongly influence the structural and optical properties of the $\mathrm{WO}_{3}$ inverse opal. Depending on the demands on wavelength region, we are able to tune the reflection peak positions of the inverse opal from $469 \mathrm{~nm}$ to $1533 \mathrm{~nm}$ by synthesizing PMMA spheres with diameters in the range of $154-647 \mathrm{~nm}$. The sphere size was modified by changing parameters such as the temperature of the reaction or the concentration of the MMA [4]. The size of the spheres is uniform (dispersity normally smaller than 0.1 ), which is crucial for the formation of the ordered structure (left SEM image in Fig. 1). Because of the possible incomplete infiltration of pores and shrinkage of precursor during thermal conversion, the number of defects in the inverse opal increases compared to the opal template (compare the left and right SEM images in Fig. 1). Additionally the size of the ordered domains of inverse opal (about $100 \mu \mathrm{m}$, see Fig. 5) is smaller than the domain size of opal films. Cracks and other defects can lead to the reduction of reflectance of the photonic crystals. Nevertheless, the measured spectra of the $\mathrm{WO}_{3}$ inverse opal films show clear reflection peaks (Fig. 2c). Position and intensity of the reflection peak can be utilized for different sensing applications.

\section{Refractive Index Senor for Fluid Detection}

Some optical properties of PhCs like the color caused by the photonic band structure allow designing straightforward and thus practical system for sensing. As an example we present a cost-effective system based on $\mathrm{WO}_{3}$ inverse opal structure for detection of fluids or their mixtures with different refractive indices [5].

Change of refractive index of the fluid in the pores of inverse opal structure leads to the change of the observed color of the PhC (Fig. 2a) which originates from the shift of the photonic band gap positions (Fig. 2b). To test the usability of this effect for the detection of different fluids static measurements were performed. Droplets of four different liquids with different refractive indices (Table 1) are placed on top of the $\mathrm{WO}_{3}$ inverse opal films and the color change was monitored using a fiberspectrometer.

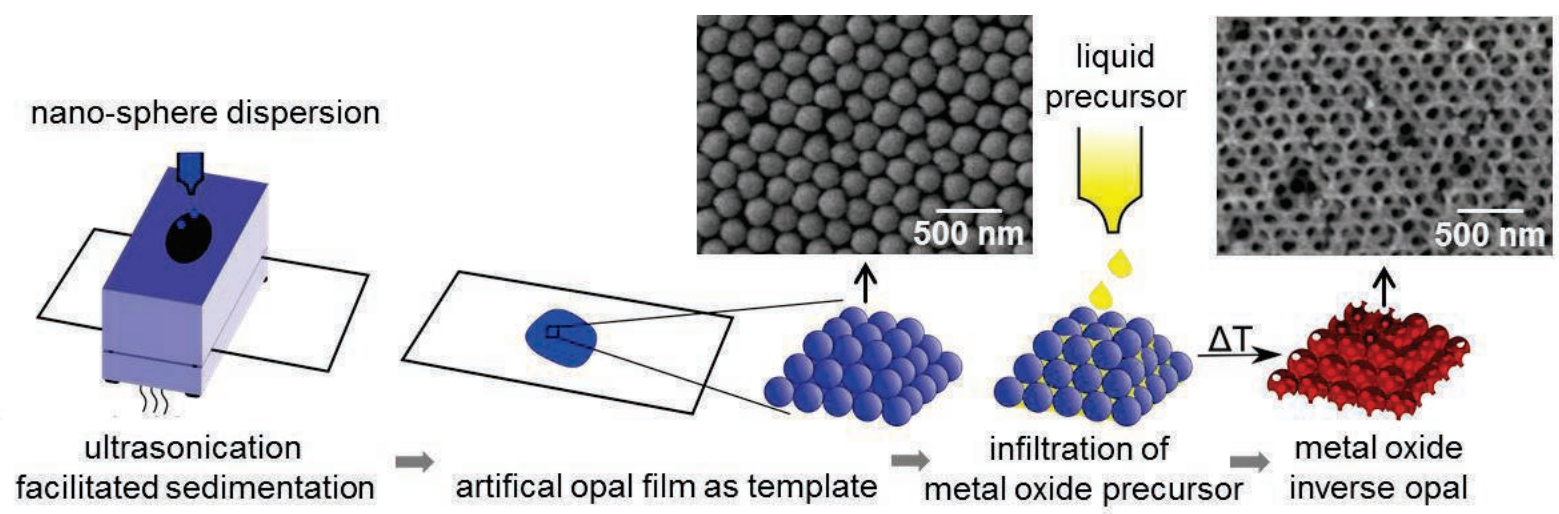

Fig. 1. Scheme of the synthetic method of metal oxide inverse opal films. The two SEM micrographs show the top surfaces of the PMMA opal structure (left) and the $\mathrm{WO}_{3}$ inverse opal structure (right). 
The resulting reflectance spectra (Fig. 2c) show that, as the refractive index of the pore fluid increases, the peak shifts to longer wavelength region. This shift is consistent with the calculated photonic band structure (Fig. 2b). If only the peak shift is taken into account it is possible to identify the fluids clearly except methanol and water (refractive index difference of 0.006). A more elaborate data analysis reveals a sensitivity of the system of 327 $\mathrm{nm} / \mathrm{RIU}$, which is in the order of the theoretically predicted value of $270 \mathrm{~nm} / \mathrm{RIU}$ [5] and comparable to other, experimentally realized sensitivities reported in literature $[7,8]$.

The calculated photonic band structures show a correlation between band position, band gap width and refractive index of the pore fluid (e.g. Fig. 2b) [5]. Additionally, under the optical consideration, it is predictable that the reflectance of the PhCs will change when different fluids are introduced into the inverse opal structure which changes the refractive index contrast between metal oxide and fluids. This small change of reflectance is also shown in the measured spectra among different fluids (Fig. 2c). Therefore, theoretically, the most efficient way to readout the optical response of this type of fluid sensor is to take all the three spectral changes (peak position, bandwidth and reflectance) into account. The experimentally approach to achieve this high sensitivity is to measure the intensity change at a certain wavelength on the rising or falling edge of the reflection peak. We therefore analyzed the reflectance change of the static measurements at the rising edge of the reflection peak (at $580 \mathrm{~nm}$ ). The limit of detection can then be estimated to be as high as 0.001 RIU [5]. However, we note that this approach can be applied only when there are no other interactions between the PhC and the fluid (e.g. change of electronic properties of $\mathrm{PhC}$ ) which can superimpose the spectral shift and if the variation in refractive index of the fluid is limited to a known range.

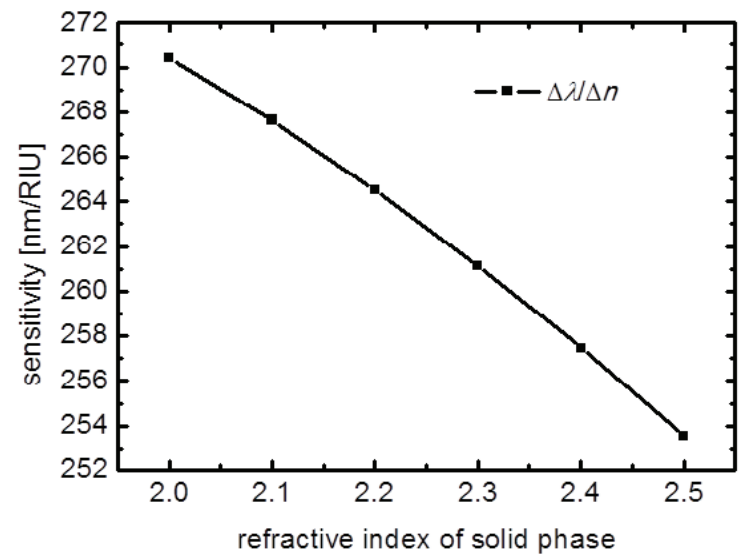

Fig. 3. Calculated sensitivity of the refractive index sensor depends on the refractive index of the constituting material in inverse opal structure.

For further optimization structural and material parameters of the $\mathrm{PhC}$ can be modified to change the sensitivity of the system.

Tab. 1: Fluids used for static measurements and their refractive indices $n$.

\begin{tabular}{lll}
\hline fluid & $n$ & reference \\
\hline methanol & 1.327 & {$[9]$} \\
water & 1.333 & {$[10]$} \\
ethanol & 1.361 & {$[11]$} \\
isopropyl alcohol & 1.379 & {$[12]$} \\
\hline
\end{tabular}

For example, increasing the lattice constant of the structure will linearly increase the spectral shift per RIU.
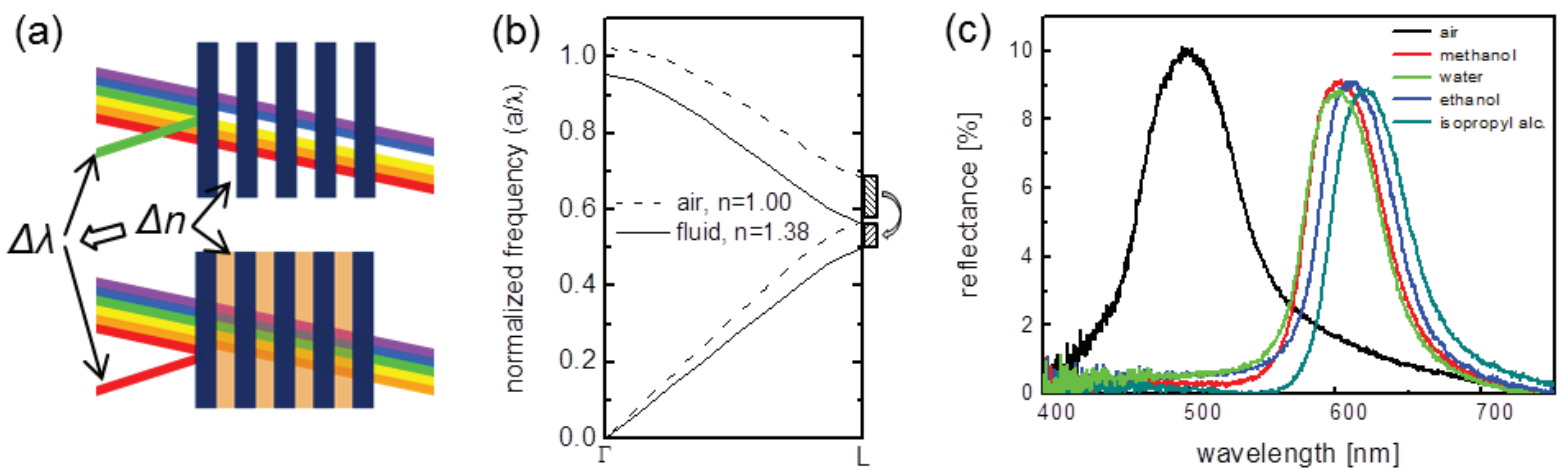

Fig. 2. (a) $1 \mathrm{D} \mathrm{PhC}$ as a model explaning the sensing mechanism of refractive index sensor for fluid detection. (b) Calculated photonic band structure of $\mathrm{WO}_{3}$ inverse opal structure along $\Gamma$ - $L$ direction in reciprocal space. The differently shadowed rectangular boxes indicate the change of band gap when the pore is filled with air (solid curves) or a fluid (dashed curves). a: lattice constant, $\lambda$ : wavlength of light, $n$ : refractive index. (c) Measured reflectance spectra of the $\mathrm{WO}_{3}$ inverse opal film (black curve) and the spectral shift when its pores are filled with liquids of different refractive indices (colored curves). 
However, scaling up the structure will also shift the operating spectral range from visible to IR range. In this study, we used $\mathrm{WO}_{3}$ as an example for metal oxide in general, but the material to form the $\mathrm{PhC}$ is of course not limited to this. To estimate the potential sensitivity increase by utilizing different oxides band structure simulations were carried out. Results show that even a change of refractive index of the material from 2.50 to 2.00 leads only to a minor improvement of sensitivity from 254 $\mathrm{nm} / \mathrm{RIU}$ to $270 \mathrm{~nm} / \mathrm{RIU}$ but at the expense of possible lower reflectance (Fig. 3). Therefore, for the design of the system, the choice of material should be more focused on the physical and chemical interaction between the fluid and the material rather than the refractive index of material.

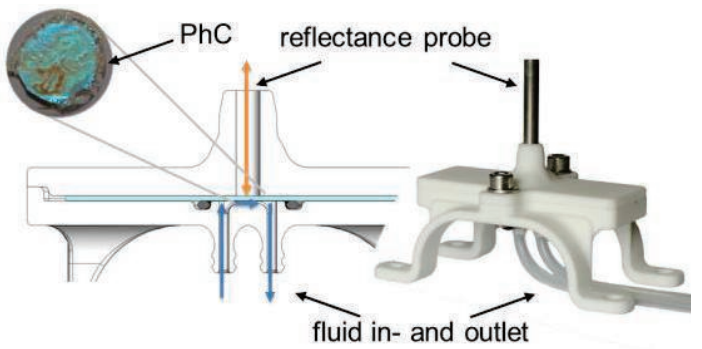

Fig. 4. Schematic drawing of the cross section of the additive manufactured sample cell (left) and a photograph of the cell (right).

The approach of measuring the intensity change at single wavelength also allows us to easily generate continuous signals without complex data evaluation, such as determination of the peak position. Therefore, we are able to perform dynamic fluid measurements to detect continuous refractive index change of fluid as a function of time. This experiment is carried out in a custom-built sample cell build by additive manufacturing technique (polymer laser sintering). The cell features three openings for the reflection probe and fluid in- and outlet (Fig. 4). A micro-pump is used to supply a stable flow. We measured the reflection intensity change of the $\mathrm{WO}_{3}$ inverse opal structure at $620 \mathrm{~nm}$ while it was continuously flushed by water/ethanol mixtures with different ethanol content from pure water $(0 \mathrm{~mL} / 5 \mathrm{~mL}$ fluid) to pure ethanol (5 mL/5 mL fluid). The measured response of the system defined as:

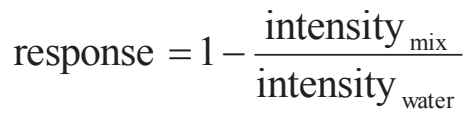

with the mean value of the intensity at a certain ethanol content (intensitymix) and the intensity at pure water (intensity water) is in good agreement with the literature data which are measured in a static experiment $[5,13]$.

\section{Thermal Stability of Inverse Opal Structure}

Another advantage of using this inverse opal structure as a sensing element is that its structural size (macroporous, thick walls) is larger compared to e.g. mesoporous sensing material typically used for semiconducting gas sensors. Consequently, it is expected to provide higher structural stability at elevated temperature and higher accessibility for gas. To test the structural stability, we annealed the $\mathrm{WO}_{3}$ inverse opal films at temperatures up to $550{ }^{\circ} \mathrm{C}$ in air for $5 \mathrm{~h}$ with a heating rate of $5^{\circ} \mathrm{C} / \mathrm{min}$. We found that, even though the synthetic method and parameters for all the samples are the same, the thermal stability of different samples varies.
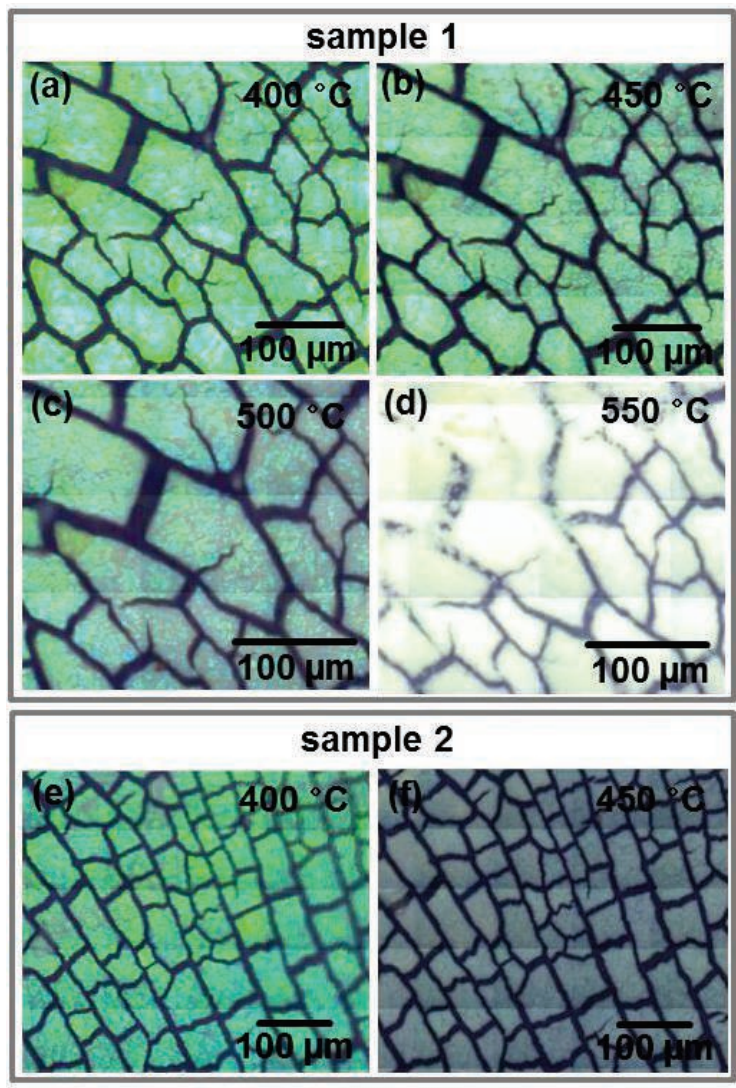

Fig. 5. Optical micrographs of two representative samples of $\mathrm{WO}_{3}$ inverse opal films heat treated at different temperatures.

Fig. 5 shows two representative samples. Sample 1 still displays green color at $500{ }^{\circ} \mathrm{C}$ (Fig. 5c) while sample 2 lost its color completely already at $450{ }^{\circ} \mathrm{C}$ (Fig. 5f). SEM micrograph of the more stable sample at $550^{\circ} \mathrm{C}$ (Fig. 6a) reveal residuals of ordered lattice structure of the original inverse opal structure among other irregular structures, whereas for the less stable sample treated at the same temperature no ordered structure is visible (Fig. 6b). The complex phase transformation of $\mathrm{WO}_{3}$ in this temperature region is held responsible for the poor reproducibility of thermal stability. Already 
for the as prepared samples (after thermal conversion at $400{ }^{\circ} \mathrm{C}$ without further annealing), we identified different mixtures of phases among samples with variations in thermal stability after further heat treatment (XRD analysis, data not shown). This preliminary work shows that even small differences during the synthetic process of $\mathrm{WO}_{3}$ inverse opal structures, such as different temperature in the furnace, can lead to reduced reproducibility of structural properties. Precise control of the synthetic process or choice of metal oxide with less complex phase transformation at application temperature will therefore be crucial for the application in high temperature.

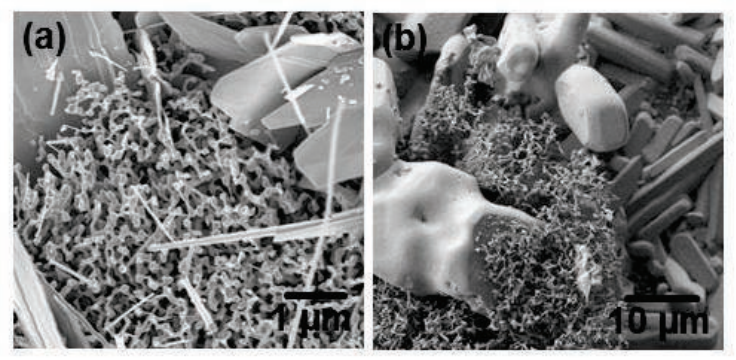

Fig. 6. SEM micrographs of the more (a) and less (b) stable $\mathrm{WO}_{3}$ inverse opal films heat treated at $550^{\circ} \mathrm{C}$.

\section{Conclusions}

Nanocasting process using opal structure template can be used to relatively easily synthesize metal oxide inverse opal structure as a photonic crystal based sensing material. Uncomplicated refractive index sensor using this material can be used to detect different fluids via the optical readout of the intensity change at single wavelength for both static and continuous flow measurements. The sensitivity of the presented system is $327 \mathrm{~nm} / \mathrm{RIU}$, and the limit of detection is estimated to be 0.001 units. Defects of the structure or moderate variations of the refractive index of the material forming the structure are not critical for the sensor operation or its sensitivity.

Metal oxides (e.g. $\mathrm{WO}_{3}$ ) inverse opal films are suitable for room temperature fluid sensing since they are chemically stable, but further work is required to optimize its thermal stability in terms of the control of the synthetic process and the choice of material.

\section{Acknowledgements}

We acknowledge the financial support by the German Federal Ministry of Education and Research (BMBF, grant no. 13N12969) and the support of the project executing organization (VDI Technologiezentrum GmbH, Düsseldorf).

\section{References}

[1] R.V. Nair, R. Vijaya, Photonic crystal sensors: An overview, Progress in Quantum Electronics 34, 89-134 (2010); doi:

10.1016/j.pquantelec.2010.01.001

[2] H. Xu, P. Wu, C. Zhu, A. Elbaz, Z.Z. Gu, Photonic crystal for gas sensing, J. Mater. Chem. C 1, 6087-6098 (2013); doi: 10.1039/C3TC30722K

[3] A. Stein, F. Li, N.R. Denny, Morphological Control in Colloidal Crystal Templating of Inverse Opals, Hierarchical Structures, and Shaped Particles, Chem. Mater. 20, 649-666 (2008); doi: $10.1021 / \mathrm{cm} 702107 \mathrm{n}$

[4] M. Egen, R. Zentel, Surfactant-Free Emulsion Polymerization of Various Methacrylates: Towards Monodisperse Colloids for Polymer Opals, Macromol. Chem. Phys. 205, 1479-1488 (2004); doi: 10.1002/macp.200400087

[5] S. Amrehn, X. Wu, C. Schumacher, T. Wagner, Photonic crystal-based fluid sensors: Toward practical application, Phys. Status Solidi A n/a (2015); doi: 10.1002/pssa.201431875

[6] M. Sasaki, K. Hane, Ultrasonically facilitated twodimensional crystallization of colloid particles, J. Appl. Phys. 80, 5427 (1996); doi: 10.1063/1.362730

[7] J. Wu, D. Day, M. Gu, A microfluidic refractive index sensor based on an integrated threedimensional photonic crystal, Appl. Phys. Lett. 92, 071108 (2008); doi: 10.1063/1.2840700

[8] B. Wang, M.A. Dündar, R. Nötzel, F. Karouta, S. $\mathrm{He}$, van der Heijden, Rob W., Photonic crystal slot nanobeam slow light waveguides for refractive index sensing, Appl. Phys. Lett. 97, 151105 (2010); doi: 10.1063/1.3497296

[9] K. Moutzouris, M. Papamichael, S. Betsis, I. Stavrakas, G. Hloupis, D. Triantis, Refractive, dispersive and thermo-optic properties of twelve organic solvents in the visible and near-infrared, Appl. Phys. B 116, 617-622 (2014); doi: $10.1007 / \mathrm{s} 00340-013-5744-3$

[10] G.M. Hale, M.R. Querry, Optical Constants of Water in the 200-nm to 200- $\mu \mathrm{m}$ Wavelength Region, Appl. Opt. 12, 555-563 (1973); doi: 10.1364/AO.12.000555

[11] S. Kedenburg, M. Vieweg, T. Gissibl, H. Giessen, Linear refractive index and absorption measurements of nonlinear optical liquids in the visible and near-infrared spectral region, Opt. Mater. Express 2, 1588-1611 (2012); doi: 10.1364/OME.2.001588

[12] W.M. Haynes, CRC handbook of chemistry and physics: A ready-reference book of chemical and physical data, 95th ed.

[13] J. Herráez, R. Belda, Refractive Indices, Densities and Excess Molar Volumes of Monoalcohols + Water, J Solution Chem 35, 1315-1328 (2006); doi: 10.1007/s10953-0069059-4 